\title{
Progress toward a laser-driven $x$-ray free-electron laser
}

Mark Wiggins, Maria Pia Anania, Enrico Brunetti, Silvia Cipiccia, Bernhard Ersfeld, Mohammad Ranaul Islam, Riju Issac, Gaurav Raj, Richard Peter Shanks, Gregory Vieux, Gregor Welsh, Allan Gillespie, Allan MacLeod, Mike Poole, and Dino Anthony Jaroszynski

Measurements of the beam quality of electrons from the ALPHA-X laser-plasma accelerator show it is close to what is required to drive a compact short-wavelength synchrotron source.

The use of $x$-ray radiation has driven the development of synchrotron sources and, more recently, x-ray free-electron lasers (FELs). These microwave-based facilities are huge and expensive, yet governments are prepared to support them (usually one per nation) because of their great value to industry, academia, and society. However, laser-wakefield accelerators (LWFAs) are now advancing to the point where compact radiation sources could be developed into a new, complementary, or even disruptive technology. In addition to reductions in scale and cost-by a factor of up to 1000-x-ray pulse durations are significantly shorter than those of conventional lasers, which could facilitate probing of ultrafast dynamic processes.

The Advanced Laser-Plasma High-Energy Accelerators towards X-rays (ALPHA-X) project, based at the University of Strathclyde, is developing laser-plasma accelerators as drivers of radiation sources. ${ }^{1}$ The first demonstration of a compact synchrotron source based on an LWFA was recently demonstrated using a conventional undulator and a diverging electron beam. ${ }^{2}$ Our challenge is to develop a compact x-ray FEL. Here we focus on developments to improve the electron-beam properties and discuss the suitability of LWFAs as drivers of FELs. Our work shows that the once-distant prospect of a compact $x$-ray FEL is now within reach.

On the ALPHA-X accelerator beam line (see Figure 1), electrons are accelerated in a relativistically self-guiding plasma channel formed in a hydrogen-gas jet by a $35 \mathrm{fs}$ titaniumsapphire laser pulse with a power of 1J. Electrons are selfinjected from the background plasma into the density wake that trails behind the laser pulse (through the combined action

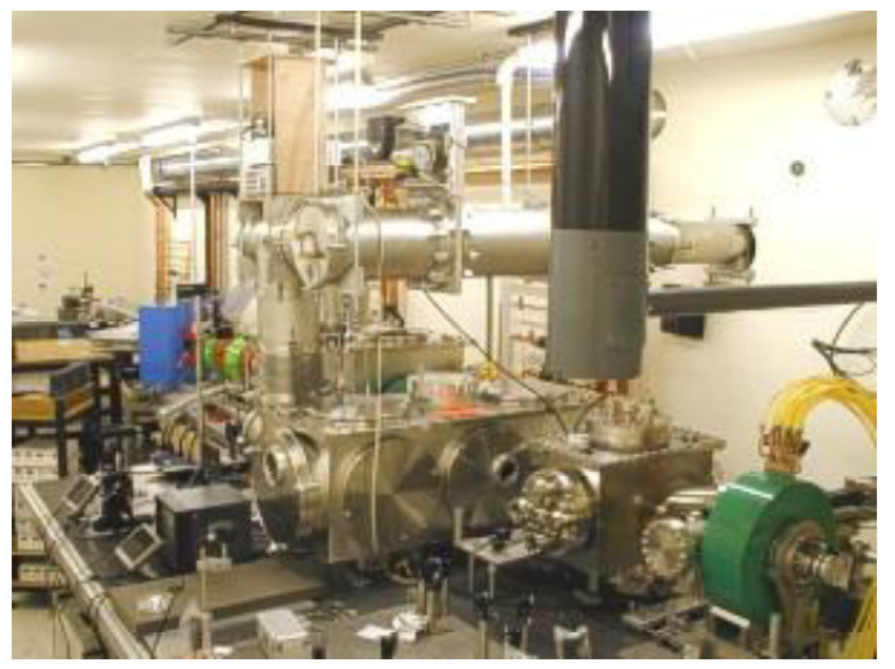

Figure 1. The ALPHA-X compact wakefield accelerator, beamtransport system, and undulator.

of the laser's ponderomotive force and the plasma's restoring force). Measurements of the electron-energy spectra are carried out using a magnetic-dipole spectrometer with a wide energy range (up to $700 \mathrm{MeV}$ ) and excellent energy resolution $(<0.5 \%)$.

To date, laser-plasma accelerators have produced electron beams with rms energy spreads typically in the $2-10 \%$ range that are usually instrument-resolution limited..$^{2-5}$ Our initial results show that monoenergetic electron beams of up to $100 \mathrm{MeV}$ can be produced with an rms relative energy spread $\sigma_{\gamma} / \gamma<0.8 \%$ (see Figure 2), where $\gamma$ is the Lorentz factor. We also demonstrate effective beam transport using dipole and quadrupole magnets. We know from simulations that the electron bunches are a fraction of a relativistic plasma wavelength (i.e., $\ll \lambda_{\mathrm{p}} \approx 6 \mu \mathrm{m}$ for our gas-jet parameters), which implies that electrons see a large variation in potential across their length. However, the Coulomb force of the electron bunch produces its own wake, which has the effect of partially cancelling out the laser-generated wake and thus of reducing the variation in the potential. This results in a

Continued on next page 


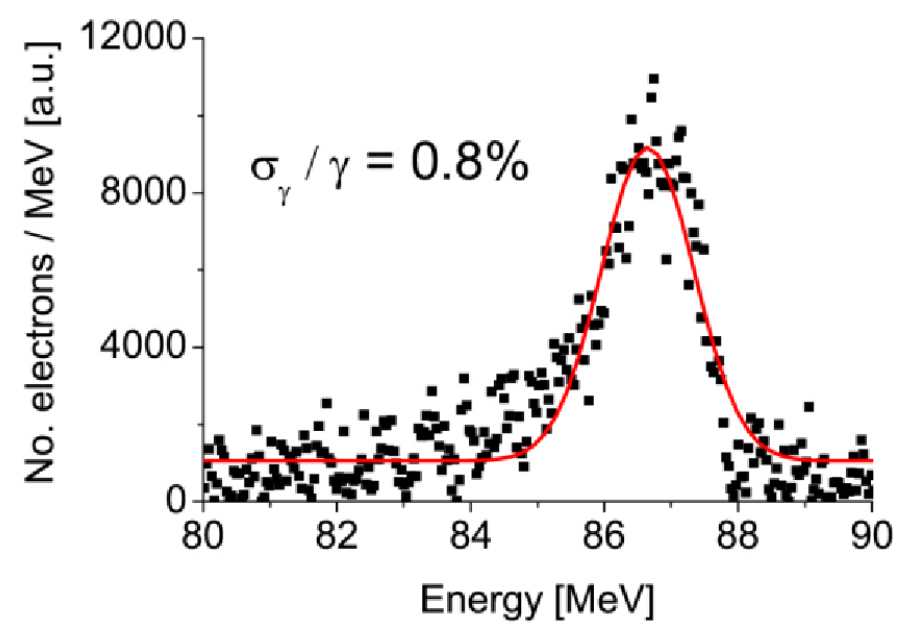

Figure 2. Electron-energy spectrum measured using a magnetic-dipole spectrometer, in arbitrary units (a.u.). $\sigma_{\gamma} / \gamma$ : rms relative energy spread.

marked reduction in the energy spread (see Figure 3).

We explore the implications for FEL operation using our $15 \mathrm{~mm}$ period-magnetic undulator. CCD images of the transverse electron-beam profile show that the divergence is of order $2 \mathrm{mrad}$. Assuming a beam size at the accelerator exit (on the basis of simulations) on the order of a few microns, we can estimate the beam's normalized emittance $\varepsilon_{n}<1 \pi \mathrm{mm}$ mrad. This satisfies one condition for FEL gain, which requires the geometric emittance to be less than the wavelength, i.e., $\varepsilon_{n}<\lambda \gamma / 4 \pi$, where $\lambda$ is the radiation's wavelength ( $\lambda \approx 250 \mathrm{~nm}$ for our setup). ${ }^{6}$

A more stringent condition exists for the energy spread. For net FEL gain, $\sigma_{\gamma} / \gamma$ should be less than the FEL gain parameter $\rho .^{6}$ For a $10 \mathrm{pC}$ electron bunch in our beam line, we estimate $\rho \approx 0.011$ and 0.002 for $\lambda=250$ and $8 \mathrm{~nm}$, respectively. Comparing this to the measured energy spread (upper limit: $\sigma_{\gamma} / \gamma=0.008$ ), the beam quality is close to (or may already satisfy) the requirement for net FEL gain.

We showed that the quality of the electron beams produced by our laser-plasma accelerator approaches that required for an FEL, which would substantially increase the photon yield. The next step is to pass the matched electron beam through an undulator to realize a compact vacuum-UV FEL. Our current setup should also be able to demonstrate FEL gain in the soft $x$-ray regime $(\lambda<10 \mathrm{~nm})$.

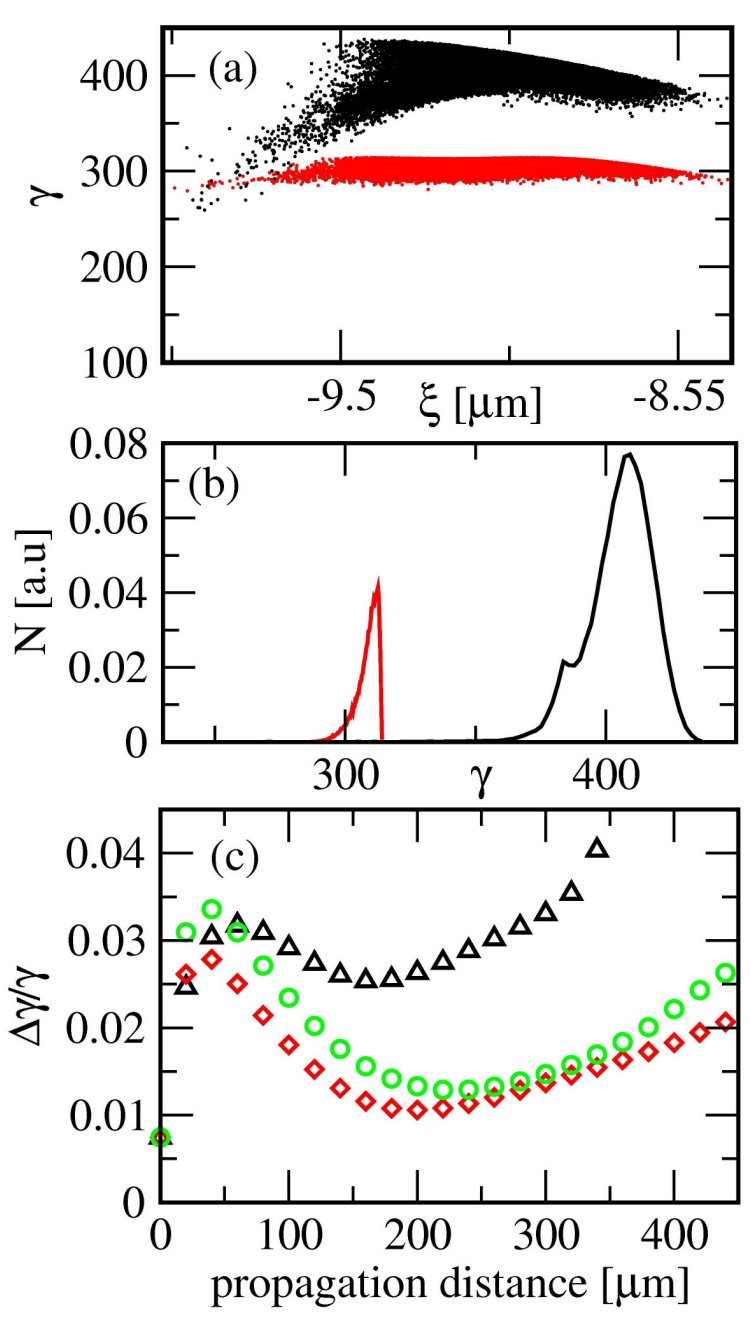

Figure 3. Simulations showing energy-spectrum compression due to beam loading. $\xi$ is an axial coordinate parameter showing that the electron bunch length is about 1 micron. $\gamma$ is the Lorentz factor.

We acknowledge support by the UK Engineering and Physical Sciences Research Council and the European Commision's New and Emerging Science and Technology Activity under the Sixth Framework Programme 'Structuring the European Research Area' program (project EuroLEAP, contract number 028514). 


\section{Author Information}

Mark Wiggins, Maria Pia Anania, Enrico Brunetti, Silvia Cipiccia, Bernhard Ersfeld, Mohammad Islam, Riju Issac, Gaurav Raj, Richard Shanks, Gregory Vieux, Gregor Welsh, and Dino Jaroszynski

Scottish Universities Physics Alliance (SUPA)

Department of Physics

University of Strathclyde

Glasgow, UK

\section{Allan Gillespie}

SUPA

Division of Electronic Engineering and Physics

University of Dundee

Dundee, UK

\section{Allan MacLeod}

School of Computing and Creative Technologies University of Abertay Dundee

Dundee, UK

\section{Mike Poole}

Accelerator Science and Technology Centre Daresbury Laboratory

Warrington, UK

\section{References}

1. D. A. Jaroszynski et al., Radiation sources based on laser-plasma interactions, Phil. Trans. R. Soc. A 364, pp. 689-710, 2006. doi:10.1098/rsta.2005.1732

2. H.-P. Schlenvoigt et al., A compact synchrotron radiation source driven by a laser plasma wakefield accelerator, Nat. Phys. 4, pp. 130-133, 2008. doi:10.1038/nphys811

3. S. P. D. Mangles et al., Monoenergetic beams of relativistic electrons from intense laserplasma interactions, Nature 431, pp. 535-538, 2004. doi:10.1038/nature02939

4. J. Faure et al., A laser-plasma accelerator producing monoenergetic electron beams, $\mathbf{N a -}$ ture 431, pp. 541-544, 2004. doi:10.1038/nature02963

5. W. P. Leemans et al., GeV electron beams from a centimetre-scale accelerator, Nat. Phys. 2, pp. 696-699, 2006. doi:10.1038/nphys418

6. R. Bonifacio, C. Pellegrini, and L. Narducci, Collective instabilities and high-gain regime in a free electron laser, Opt. Commun. 50, pp. 373-378, 1984. doi:10.1016/00304018(84)90105-6 